\title{
Propiedades electrónicas e interacciones magnéticas en Sistemas Modelo de Metaloproteínas
}

\author{
Nicolás Ignacio Neuman \\ niconeuman@gmail.com \\ Dr. Carlos D. Brondino \\ Dr. Alberto C. Rizzi \\ Departamento de Física \\ Facultad de Bioquímica y Ciencias Biológicas \\ Universidad Nacional del Litoral \\ Fecha de la defensa: 22/03/2013
}

\section{Resumen}

En este trabajo de tesis estudiamos las propiedades espectroscópicas y magnéticas de cuatro compuestos con metales de transición paramagnéticos, dos magnéticamente concentrados y dos diluídos: [Cu(II) (tda)(phen) $]_{2} \cdot \mathrm{H}_{2}$ tda (tda $=$ tiodiacetato, phen $=1,10$-fenantrolina) (Cu(tda)phen) y $\left[\mathrm{Zn}(\mathrm{II})_{1-\mathrm{x}} \mathrm{Cu}(\mathrm{II})_{x} \text { (tda)phen }\right]_{2} \cdot \mathrm{H}_{2}$ tda $(x \approx 0.2$, $0.12,0.1$ y 0.03$)(\mathrm{Zn}(\mathrm{Cu})$ (tda)phen), catena$\mathrm{Co}(\mathrm{II})$ (Fumarato) $\left(\mathrm{H}_{2} \mathrm{O}\right)_{4}$ (Co(II)Fumarato) y catena-Zn(II) ${ }_{1-y} \mathrm{Co}(\mathrm{II})_{y}$ (Fumarato) $\left(\mathrm{H}_{2} \mathrm{O}\right)_{4}(\mathrm{y} \approx$ $0.1,0.05$ y 0.03 ) (Zn(II)Co(II)Fumarato).

Los estudios de EPR realizados en el compuesto dimérico $\mathrm{Cu}($ tda)phen y su análogo isostructural de $\mathrm{Zn}(\mathrm{II})$ dopado con $\mathrm{Cu}$ (II) nos permitieron determinar los parámetros magnéticos correspondientes a los iones $\mathrm{Cu}(\mathrm{II})$ que componen las unidades diméricas y la interacción magnética entre ellos. El análisis de los espectros de monocristal en el sistema magnéticamente concentrado utilizando el modelo de colapso por intercambio de Anderson extendido por nosotros para considerar poblaciones de dímeros con vecinos en distintos estados magnéticos, nos permitió determinar la magnitud de las interacciones interdiméricas asociadas a interacciones $\pi-\pi$ entre los ligandos 1,10-fenantrolina.

Los estudios realizados en Co(II)Fumarato y $\mathrm{Zn}(\mathrm{II}) \mathrm{Co}(\mathrm{II})$ Fumarato nos permitieron determinar los parámetros magnéticos asociados a los iones Co(II) en un entorno octahédrico distorsionado. El análisis de resultados de mediciones magnéticas y de EPR en los compuestos magnéticamente concentrado y diluido nos permitió determinar las interacciones magnéticas entre iones Co(II) asociadas a caminos químicos que involucran puentes de $\mathrm{H}$ o el ligando fumarato. Además pudimos establecer correlaciones entre los parámetros magnéticos y el entorno de coordinación, las cuales interpretamos a partir de cálculos basados en la teoría de campo cristalino, y entre los acoplamientos magnéticos y los caminos químicos que conectan los iones metálicos.

Electronic Properties and Magnetic Interactions in Model Systems of Metalloproteins

\section{Summary}

This thesis work involves the study of four compounds of paramagnetic transition metal ions: $\left[\mathrm{Cu}(\mathrm{II})(\mathrm{tda})(\text { phen) }]_{2} \cdot \mathrm{H}_{2}\right.$ tda (tda $=$ thiodiacetato, phen $=1,10$-phenanthroline) (Cu(tda)phen) and $\left[\mathrm{Zn}(\mathrm{II})_{1-x} \mathrm{Cu}(\mathrm{II})_{x}(\mathrm{tda})\right.$ phen $]_{2} \cdot \mathrm{H}_{2}$ tda $(x \approx 0.2,0.12,0.1$ and 0.03 ) ( $\mathrm{Zn}(\mathrm{Cu})(\mathrm{tda})$ phen), catena-Co(II)(Fumarato) $\left(\mathrm{H}_{2} \mathrm{O}\right)_{4}(\mathrm{Co}(\mathrm{II})$ Fumarato $)$ and catena-Zn(II) ${ }_{y} \mathrm{Co}(\mathrm{II})_{y}$ (Fumarato) $\left(\mathrm{H}_{2} \mathrm{O}\right)_{4}(y \approx 0.1,0.05$ and 0.03) (Zn(II)Co(II)Fumarato). 
EPR studies of the dimeric compound $\mathrm{Cu}($ tda)phen and its $\mathrm{Zn}(\mathrm{II})$ isostructural analog doped with $\mathrm{Cu}(\mathrm{II})$ allowed us to determine the magnetic parameters of the $\mathrm{Cu}(\mathrm{II})$ ions which compose the dimeric units, as well as the magnetic interaction between them. Analysis of the single-crystal spectra in the magnetically concentrated system using Anderson's exchange narrowing model, extendend by us to consider dimer populations with neighbors in different magnetic states, allowed us to determine the magnitude of interdimeric interactions associated with $\pi-\pi$ interactions between phenanthroline ligands.

Studies of $\mathrm{Co}(\mathrm{II})$ Fumarato and $\mathrm{Zn}(\mathrm{II}) \mathrm{Co}(\mathrm{II})$
Fumarato allowed us to determine the magnetic parameters associated with Co(II) ions in a distorted octahedral environment. Analysis of magnetic measurements and EPR results in magnetically concentrated and diluted samples allowed us to determine magnetic interactions between Co(II) ions associated with chemical paths which involve Hydrogen bonds or the fumarate anion ligand. Also we established correlations between magnetic parameters and the coordination environment, which we interpreted performing calculations based on the crystal field theory, and between magnetic couplings and the chemical paths which connect the metallic ions.

\section{Implicancias de la arquitectura en la regeneración vegetativa de tres malezas perennes tolerantes a glifosato con diferentes formas de crecimiento}

\section{Elisa Panigo \\ epanigo@fca.unl.edu.ar \\ Dra. Mariel G. Perreta \\ Dr. Abelardo C. Vegetti \\ Cátedra de Morfología Vegetal \\ Facultad de Ciencias Agrarias \\ Universidad Nacional del Litoral \\ Fecha de la defensa: 26/03/2013}

\section{Resumen}

El objetivo fue identificar los caracteres morfológicos que facilitan la regeneración vegetativa de tres malezas tolerantes a glifosato: Commelina erecta L., Eustachys retusa Lag. Kunth. y Alternanthera philoxeroides Mart. Griseb., en los sistemas de producción con mínima labranza y uso intensivo de glifosato; y predecir su comportamiento. Primero se determinó la arqui- tectura del sistema de vástagos aéreos de las tres especies y su evolución desde la germinación. Luego se evaluaron las variaciones morfológicas, de crecimiento, bioquímicas y arquitecturales post-aplicación de glifosato en las tres especies, para identificar las estrategias que facilitan su regeneración y persistencia a campo. Se trabajó con 2 dosis de herbicida (letal y subletal) en cada especie. Debido a los diferentes niveles de susceptibilidad al herbicida que presentan estas tres malezas, las dosis para cada especie se seleccionaron a partir de un ensayo de dosis-respuesta. Por último, se analizó la presencia y ubicación de zonas meristemáticas de $C$. erecta y $E$. retusa para evaluar el rol que juegan en la regeneración vegetativa post-aplicación.

A partir del análisis arquitectural se iden- 